\author{
Daniel R. Brooks
}

\title{
ENTROPY, INFORMATION AND EVOLVING BIOLOGICAL SYSTEMS
}

\section{ABSTRACT}

Internal production rules in biological systems require outside energy but are also highly insensitive to the conditions of the external environment from which the energy comes. This leads to the production of historically constrained, spontaneously stable, complex structure. Because the production rules are physically encoded in the structure of the system, biological systems are physical information systems, and their expected behavior over time follows a general entropie dynamic. The autonomy of the production rules leads to an explanation for the reality of natural selection that does not rely on analogy with human economic theory. The historical nature of the elements of diversity at any given time leads to an expectation that the details of responses to external evolutionary forces, such as natural selection, competition, or geological changes, will be highly individualized. Hence, evolutionary regularities will tend to be highly generalized (macroevolutionary) or statistical in nature.

\section{INTRODUCTION}

Biology is a relatively young scientific discipline, having produced its first general theoretical formulations only in the latter half of the nineteenth century. Today, most biologists accept the proposition that evolution is the unifying concept of biology. Now, in the waning years of the twentieth century, it is

32 possible that biology stands poised to attempt its first theoretical integration with other natural sciences. 1 think this is a healthy development for biology as well as for its older sibling branches of natural science. However, differences in language, emphasis, and world view will make the job of integration difficult. People of good will on all sides must cooperate in order to make this happen.

Much of the mathematical development of evolutionary theory has been based on the assumption that biological systems tend to exist in stable equilibria with, and determined by, their surroundings. This has led to many valuable insights, but such "'equilibrium thinking" may leave key elements of the existence, behavior, and evolution of biological 
systems unexplained. I believe that this is one reason we have not yet achieved a general theory of biological evolution, and in this contribution I wish to outline one approach to integrating "nonequilibrium thinking" into general evolutionary biology. By trying to establish communication between different research traditions, I hope that mydiscussion will not seem unnecessarily abstract to biologists or too naive to scientists outside biology.

Explanations of dynamic systems distinguish "forces", which act on the system, and "flows", which signify the way in which the system responds to the forces. In equilibrium systems, the distinction between forces and flows is so marked that we can equate them with "cause" and "effect" or "environment" and "system", respectively. Second, any system that is in equilibrium with its surroundings is assumed to be "at rest" or inactive with respect to the interplay of forces and flows. Hence, any changes in the surroundings will cause changes in the system. Finally, the properties of the system itself play no role in determining the equilibrium state; that state is determined by environmental conditions (the forces impinging on the system). Thus, the system is expected to assume the same equilibrium state for any given environmental configuration, regardless of the temporal sequence in which that environmental configuration arises. If the environment changes from state "A" to state "B", then back to state "A", an equilibrium system will find itself in the same state in which it began. Many components of biological systems appear to behave in this manner. However, there are important aspects of biological systems that violate these assumptions, ranging from metabolic reactions to cell division and ontogeny, to reproduction and death, and finally to speciation and extinction.

If biological systems are not equilibrium systems, what kind of "nonequilibrium systems" are they? There are at least two general classes of nonequilibrium systems. The "close to equilibrium" class of systems comprises large systems made up of a large number of subunits, or "cells". Each of these cells is assumed to exist at (or extremely close to) equilibrium. However, the system of which the cells are a part may be large enough to encompass an environmental gradient in which groups of cells are in equilibrium with environmental conditions that are different enough that there is no overall equilibrium state for the system. In the close to equilibrium idealization, the assumption of local equilibrium replaces the assumption of global equilibrium inherent in equilibrium idealizations. However, the equilibrium system assumption that "forces" are properties of the environment and "flows" are properties of the system, along with the assumption of temporal reversibility, still holds for close to equilibrium cases.

In "far from equilibrium" idealizations, there need be no assumption of local equilibrium on any scale. Rather, it is assumed that the system will adopt local "steady states", defined as the most efficient functional states possible given particular combinations of environmental and system properties at any given time. This is analogous to saying that mechanical systems follow the line of least resistance. There is no assumption that forces and flows are distinct from one another: both can be properties of the environment and of the system. Systems for which forces and flows can be considered distinct are also called 'linear" systems; consequently, far from equilibrium systems are called "non-linear". Because the system's properties may act in part as "forces" determining the "'flow", there is no reason to assume that the system will respond to the 
same environmental conditions in the same way at different times. Thus, the history of the system plays a role in determining the response to environmental forces, eliminiating assumptions of temporal reversibility.

\section{TIME}

Some physical processes are tied to the directional nature of time, others are not. For example, if you leave a pan of hot water on a table in a cold room, the water will eventually cool to room temperature. However, the reverse process, a pan of cold water spentaneously becoming warmer, never occurs. The flo w of heat between the water and the surrounding air thus incorporates a directional component into the processes involved in heat transfer. Such processes are time-dependent, temporally asymmetrical or irreversible. Now consider the bonds forming among the water molecules in our hypothetical pan of water. Although the system as a whole maintains its functional integrity, the bonds which confer such a particular nature upon water are constantly breaking and reforming. Thus, any two molecules may be joined at one moment in time and separated at another, and this continues indefinitely with no general trend towards molecules being "'bonded" or being "'free". This is an example of a time-independent, temporally symmetrical or reversible process.

Biological processes show evidence of the differential influences of time. For example, suppose you were shown a film of light colored moths fluttering about in a forest of predominantly light colored trees changing to darker versions of the same moth fluttering about in a forest of darker colored trees. Since you could identify the moths as variants of the same species, and could identify an independent environmental variable correlated with the change in proportions of 
color variants, you could postulate that natural selection had occurred; however, you could not tell whether the film had been shown forwards or backwards. Natural selection is reversible through time. In contrast to this, there are many biological processes, such as reproduction, development (ontogenesis), death, speciation (phylogenesis), and extinction, that are inherently irreversible phenomena. Evolutionary change is therefore the result of a complex interaction among both reversible and irreversible biological processes.

There are many kinds of temporally asymmetrical processes. Consider the differences between the evolution of stars and the evolution of biological systems inhabiting the planets surrounding those stars. The "evolution" of different kinds of stars incorporates time-dependent regularity without historical connections. For example, the ontogeny of "BO" stars appears to have been the same for every member of that star class; however these parallels are due only to similar initial conditions and causes, not to a shared history. In other words, all BO stars are formed independently from one another, and none of them share a common ancestor that displayed the mass, luminosity and spectral characteristics that define a BO star. Rather, this star type is formed when particular initial conditions in the prestellar developmental stage are realized. Other conditions, such as insufficient mass of prestellar gas, would lead to the formation of a different class of star. In contrast to stellar evolution, bilogical evolution is dependent upon both initial conditions and the interplay of events unique to the particular history of the evolving biological system. For example, many of the similarities shared by species are the result of common ancestry and not the realization of a repeatable series of events originating from independent, but identical, initial conditions. Such similarities (homologies) are embedded within an inherited (i.e., historical) matrix. Unlike many time-dependent physical systems, bilogical systems retain many of the effects of history as these events accumulate and are transmitted from ancestor to descendants. The unfolding of a biological system's time-dependent behavior is thus constrained by the influence of the historical burden it is carrying into the future.

\section{ENTROPY}

Lotka $(1913,1925)$ was among the first 20th century authors to discuss biological systems in terms of energy flows and energy partitioning. He recognized that living systems persist in space and time by transforming energy from one state to another in a manner that generates organized structure. There are two classes of such energy transformations. The first class, heat-generating transformations, involves a net loss of energy, measured as heat, from the system. The second class, conservative transformatins, involves changing free energy into states that can be stored and utilized in subsequent transformations (Brooks, Collier, Maurer, Smith and Wiley, 1989). Although all conservative tran- 
sformations in biological systems are coupled with heat-generating transformations the reverse is not necessarily true; therefore, there is a heavy cost to maintaining structure. Lotka (1913) suggested that the inevitable structural decay which must accompany such costs could be delayed by the system's accumulation of bound energy. According to this view then, the interplay between energy flow and partitioning in biological systems acts only to slow the rate at which energy stored by conservative transformations is degraded by heat- -generating processes.

\section{TERMINOLOGY}

Energy flows within biological systems are coupled with the production of entropy. The following five points are relevant to this discussion: (1) Heat generating transformations produce thermal entropy, a measure of the tendency of the system to move towards disorganization-, (2) Conservative transformations produce structural entropy, $a$ measure of the tendency of the system to move toward'; structural complexity, (3) Dissipative structures (Prigogine, 1980) are systems in which structural entropy is produced by dissipative processes that allow a higher rate of structural entropy production than if the processes were completely thermal (heat generating); (4) because energy stored by conservative transformations degrades at a rate slower than the heat liberated during heat producing transformations, there is a period of time during which the system accumulates structural entropy. This time lag allows processes occurring within the system to be isolated from processes occurring outside the system ("phase separation"). Consequently, fluctuations in processes occurring outside the system, that could lead to disorder, are prohibited from disrupting the internal structure of the system; and (5) the formation of a phase separation between the system and its surroundings allows the evolution of internal production rules that are not governed directly by fluxes from the environment, but rather by entropy production within the system. In general, than, dissipative structures can arise kinetically when the internal dynamics of the system faster than it can equilibrate with its surroundings. ,They can also arise physically, when the boundary conditions are such that there is a physical barrier between the system and its surroundings. The greater the phase separation, or distinction between system and surroundings, the greater the autonomy of the internal production rules. For example, cell membranes are maintained kinetically and produce a physical phase separation between the living system and its environment. Hence, biological systems behave as dissipative structures, at least in some aspects or on some spatial and temporal scales.

Entropy changes (dS) in such systems can be subdivided into two components, onemeasuring exchanges between the system and its surroundings $\left(\mathrm{d}_{\mathrm{e}} \mathrm{S}\right)$ 
36 and the other measuring production by irreversible processes internal to the system $\left(\mathrm{d}_{\mathrm{i}} \mathrm{S}\right)$. Exchanges between biological systems and their surroundings are accompanied by a great deal of waste; hence, $\mathrm{d}_{\mathrm{e}} \mathrm{S}$ is very large compared to $\mathrm{d} ; \mathrm{S}$. However, if biological sysems are to maintain their structural integrity, they must produce entropy internally $\left(\mathrm{d}_{\mathrm{i}} \mathrm{S}>\right.$ $0)$. Therefore, it is $d_{\mathrm{i}} S$ that is important in considerations of biological evolution.

Production rules in biological systems are those processes for which there is an energetic "cost" or "'allocation". Following Prigogine and Wiame (1946) and Zotin and co-workers (e.g., Zotin and Zotina, 1978), Brooks and Wiley (1988)

\section{INFORMATION}

A key element in the Brooks-Wiley theory is the manner in which the concept of denoted such allocations using the symbol $\psi$, signifying a specific dissipation function. $\psi$ includes at least two classes of processes: (1) those involved in dissipation from the system, called the external dissipation function $\left(\psi_{\alpha}\right.$, or thermal entropy) and (2) those involved in dissipation within the system, called the bound dissipation function $\left(\psi_{\mu}\right)$. In biological systems, $\psi_{\mu}$ can be further subdivided into allocations for accumulating biomass $\left(\psi_{\mu}^{b}\right)$ and allocations for accumulating genetic diversity $\left(\psi_{\mu}{ }^{i}\right)$. Brooks and Wiley (1988) suggested that all three components of the biological production term $\psi$ should be included in the thermodynamic production term $d_{i} S$, shown heuristically as

$$
\mathrm{d}_{\mathrm{i}} \mathrm{S}=\psi_{\alpha}+\psi_{\mu}^{\mathrm{b}}+\psi_{\mu}^{\mathrm{i}}
$$

Biological systems must therefore have the following properties: (1) the rules (although not necessarily the details) for both heatgenerating and conservative transformations must be encoded in the structure of the system, (2) those production rules must include "information" or "instructions" leading to non-random exchanges between the system and its surroundings, and (3) production by the conservative processes must be positively entropie. Under this view, there is an entropie drive within biological systems resulting from production, which includes processes that result in the accumulation of bound energy. Since the non-random nature of this accumulation results in the production of non-random mechanical and chemical gradients within biological systems, the flow of free energy and of structural entropy occur in the same, not different, directions.

information is related both to biological systems and to entropie phenomena. Information theory has been developed from two perspectives, "communications theory" and "'physical measurement theory". Common to both of these perspectives is (1) the view that information is anything transmitted from 
a "source" through a "channel", which may have varying degrees of "noise" in it, to a "receiver" and (2) the view that information is an abstraction rather than a material part of any system.

In classical communications theory, the amount of information sent from a source is calculated using a statistical entropy function. Errors in transmission can result from poor encoding at the source or from noise in the channel. The meaningful information is that subset of the information transmitted which is actually recorded by the receiver.This makes the communications theory form of information non-physical, because the entropy of the message is maximal at the source, and the effects of all processes on the information as it is being transmitted and received is to decrease the entropy of the message. Hence, either information transmission is not a physical process, or the communications theory view of entropy is a non-physical one, because physical entropies are expected to increase as a result of work done on the system.

Physical measurement theory has provided a second formalism for information. Brillouin (1962) distingushed between "free information" which is an abstraction involved in descriptive exercises, and 'bound information", which refers to material properties of systems (but stops short of stating that information per se can be a material part of a system). Bound information is determined with respect to the complexions (microstates) of the physical system. Hence, bound information is also calculated using a statistical entropy function, but, contrary to communications theory is expected to exist only in systems for which there is a non-arbitrary microstate/macrostate distinction [i.e., $\mathrm{I}(\mathrm{M})=$ $f\left(M_{m}\right)$ ]. Bound information is defined as where $H_{\max }$ corresponds to the totally relaxed state of the system (generally estimated by a randomization of the observed components of the system at hand). Brillouin defined "I" as negentropy, which is converted into bound information by measurement (measuring devices are thus receivers), so negentropy = information. Information has a physical basis, but is not a material part of the system (however, it does point to material regularities that require explanation).

Biological information functions both as a communications system and as a physiochemical system. Thus, biologists tend to think of biological information as both material and meaningful. In addition, for an adequate account of biological evolution, we need a general expectation of the growth of information through time, rather than of a distillation of information from an initial pool of all possibilities. Evolution makes sense as an informationally negentropic process only if all genetic possibilities were present at the beginning of life, and we have simply seen a distillation and reduction in those possibilities over time, 
resulting in the diversity we see. However, bilogists do not think that evolution works that way-they believe that evolution results in the growth of information and complexity through time. So, what we need is an account of biological information that is physically realistic, that is intrinsic to the system rather than to measuring devices (i.e. it is material rather than abstract), and which can grow over time as a result of spontaneous (entropie) processes.

\section{IS THERE A PHYSICAL BASIS FOR BIOLOGICAL INFORMATION?}

Intrinsic information is related to concepts of the „causal capacity” of a system, or its ability to impose distinctions on its surroundings (including measuring devices). Hence, the emphasis is an how the system produces affectc on measuring devices and not on how the measuring devices affect the system. Physical information systems (those having intrinsic information) occur as arrays, or multidimensional messages, in which microstate and macrostate distinctions are distinguished non-arbitrarily (Collier, 1987; see also Brooks, Collier, Maurer, Smith, and Wiley, 1989). Collier (1987) stated that three conditions must be met in order for the Brooks-Wiley view of information to be related to physical concepts there must be (a) an energetic "cost" in producing and maintaining biological information, (b) a physical (material) basis for the information, and (c) a real (i.e., non-arbitrary) macrostate/microstate distinction.

According to Collier's formalism, energy coming into a system can have two fates; either it can be dissipated from the system as a result of work done within

the system $\left[\psi_{\alpha}\right.$ of $\mathrm{d}_{\mathrm{i}} \mathrm{S}$, intropy (internal entropy, or the entropy produced within the system as required by nonequilibrium thermodynamics), or heat-generating processes], or it can be converted into structure within the system $\left[\psi_{\mu}\right.$ of $d_{i} S$, enformation (encoded information in the system), or conservative processes]. All conservative processes within biological systems are coupled with heat-generating processes; hence, there is a demonstrable energetic cost associated with the production and maintenance of biological information. Intropy and enformation are interconvertable (e.g., energy brought in from outside can be converted into structure, say glycogen, and that structure can be converted into heat). Intropy is converted into enformation by cohesive properties of the system. Cohesion is thus analogous to friction, which slows dissipation in purely energetic systems. Cohesive properties, which can range from molecular affinities to cell-cell adhesion to genetic compatibility, mate recognition, and genealogy, also provide resistance to fluctuations from lower levels, and this is a key to (a) the microstate/macrostate distinction, (b) the origin of natural selection, and (c) the emergence of hierarchical structure in biological systems.

Under Collier's view, microstate/macrostate distinctions are determined by part/whole associations. For example, a protein coding unit might be considered 
a macrostate, while all the actual sequences that code for that protein would constitute the microstates. Or, one could consider a locus to be a macrostate, and all sequences that correspond to that locus to be microstates. Extending the reasoning further, phenotypes could be macrostates, and all genotypes corresponding to a given phenotype could be microstates. What makes all this difficult for standard views of evolutionary biology is that the encoded information is also the carrier of the cohesive properties, so production of biological information involves the production of variation and constraints at the same time, and this alone ensures that genealogy will be a combination of continuity and change. Thus, the Brooks-Wiley theory is radical in stating that genealogical processes alone are necessary and sufficient for evolution to occur. That position does not rule out a significant role for natural selection in the production of any actual evolutionary sequence, but it does call into question its exact role.

\section{THE SHAPE OF BIOLOGICAL EVOLUTION}

Frautschi (1988; see alsoLayzer, 1975;Frautschi, 1982; Landsberg, 1984 a, b) recently contrasted two classes of processes that generate entropy. The first is equilibration of temperatures between system and surroundings; for open systems this comes through heatgenerating transformations. Bilogical systems exhibit this kind of entropie behavior through external dissipation processes ${ }^{i}\left(\psi_{\Theta}\right)$. The second is expansion of the phase space occupied by the system; an increase in its number of accessible microstates (possible configurations). System organization increases so long as equilibration (equiprobable distribution of the system over all of its microstates) take longer than phase space expansion, allowing a lag between the increase in realized entropy $\left(\mathrm{H}_{\mathrm{obs}}\right.$, or "complexity") and the increase in maximum possible entropy $\left(\mathrm{H}_{\max }\right)$ (fig. 1; for biological applications see Ulanowicz, 1980; Brooks, LeBlond and Cumming, 1984; Brooks, Cumming and LeBlond, 1988; Smith, 1988). In other words, so long as the phase space expands faster than the system can fill it up, conservative processes will be an allowed class of entropyproducing phenomena. In cosmology, this argument is used to explain the spontaneous and irreversible formation of stars, solar systems, galaxies and other organized structures, in which gravity slows down the entropie expansion of matter in the universe to such an extent that organized structures can emerge even though entropy is increasing. In biological systems, mutations act to expand the genetic phase space (Layzer, 1978, 1980) while genetic and genealogical bonds linking all organisms play an analogous role to gravity.

The increase in the number of accessible microstates is accomplished by the production of new components, either at a given organizational level or through the opening up of new levels. Free energy and structural entropy may accumulate together in such systems. In biological systems this is accomplished by 
conservative transformations. For example, auto-catalytic processes producing monomers make "monomer space" available for chemical evolution. Some monomers have high chemical affinities for each other, and will spontaneously clump into dimers and polymers. Once polymers begin to form, "'polymer space" becomes available to the evolving system. At this level, polymers are "macrostates" and monomer and dimer distributions are the "microstates". Causal interactions among polymers create new levels of organization in which polymer distributions are the microstates and new levels of oreanizaton are macrostates.

and so on. Because new levels create a hierarchy of increasing structural intricacy (= complexity + organization), and more and more of the entropy production is invested in structure, the allocation of $d_{i} S$ to $\psi_{\mu}$ should be proportional to entropy increases due to expansion of phase space.

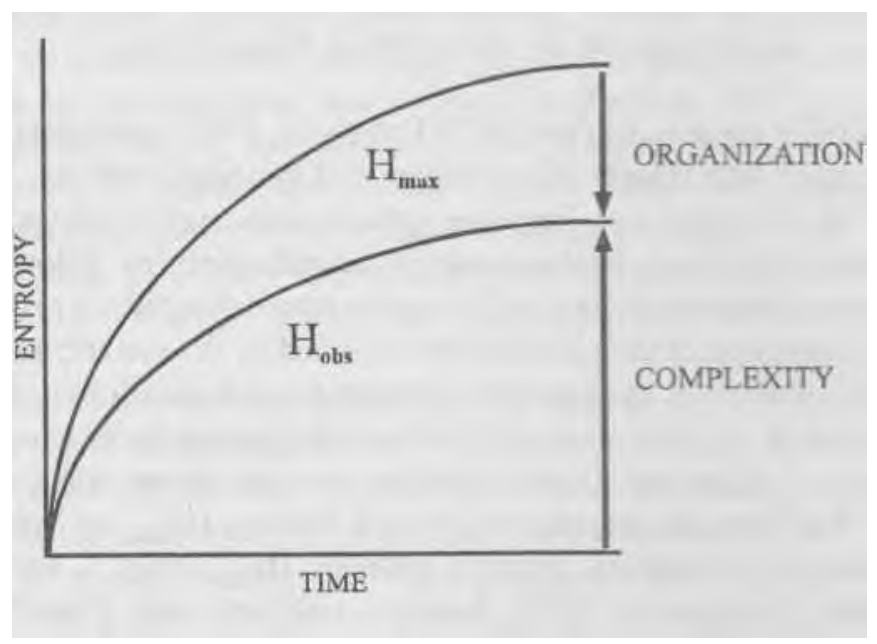

Fig. 1. The relationship between an increasing entropy maximum $\left(\mathrm{H}_{\max }\right)$ and the observed entropy $\left(\mathrm{H}_{\mathrm{obs}}\right)$ of a physical system over time. The value of is a measure of the realized entropy (or complexity) of the system, which is expected to increase over lime in accordance with the second law of thermodynamics (denoted by the upward pointing arrow). The difference between $\mathrm{H}_{\max }$ and $\mathrm{H}_{\mathrm{obs}}$ is proportional to the organization (or structure) within the system. In biological evolution, this difference is expected to increase as a result of the historical accumulation of constraints that retard the increase in realized entropy (denoted by the downward pointing arrow).

The difference between the entropy maximum $\left(\mathrm{H}_{\max }\right)$ and the actual entropy $\left(\mathrm{H}_{\mathrm{obs}}\right)$ is proportional to the organization of the system at that level (fig. 1). This difference has also been referred to as the macroscopic information of the system (Layzer, 1975). According to this perspective, $\mathrm{H}_{\max }$ represents the total infor- 
mation capacity and $\mathrm{H}_{\mathrm{obs}}$ represents the information content of a physical information system. The difference between total information capacity and information content is proportional to the constraints placed on the information system (see also Brillouin, 1962; Gatlin, 1972; Brooks, LeBlond and Cumming, 1984; Collier, 1986; Brooks, Cumming and LeBlond, 1988; Smith, 1988; Brooks, Collier, Maurer, Smith, and Wiley, 1989). Overall, then, $\mathrm{H}_{\mathrm{obs}}$ (fig. 1 ) is a measure of the realized entropy as manifested by the complexity (the information

diversity) of the system. The difference between $\mathrm{H}_{\max }$ and $\mathrm{H}_{\text {obs }}$ (fig. 1) is a measure of the internal entropy $\left(\psi_{\mu}\right.$, the bound dissipation) manifested in the structural organization (the macroscopic information, or the constraints) of the biological system. From this we can see that biological information is the carrier of constraints on the system, and constraints, in turn, represent possible variation that has been historically excluded (fig. 2).

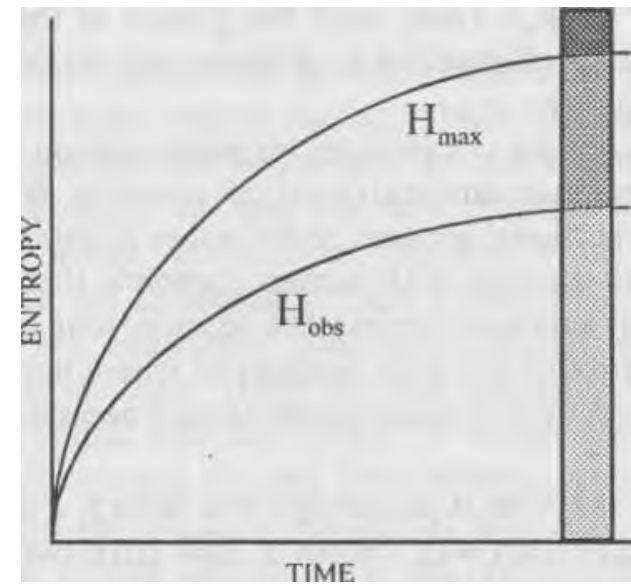

impossibl

e

historically

excluded

historically

realized

Fig. 2. The relationship between total information capacity $\left(\mathrm{H}_{\max }\right)$ and information content $\left(\mathrm{H}_{\mathrm{obs}}\right)$ of an array of physical systems comprising a number of evolutionary lineages. Historically realized diversity is measured by $\mathrm{H}_{\mathrm{obs}}$ Historical exclusion of the expression of certain kinds of informalin is proportional to $\mathrm{H}_{\max }-\mathrm{H}_{\mathrm{obs}}$ The area above $\mathrm{H}_{\max }$ represents impossible combinations at any given time.

In summary, the following three conditions form the boundary rules within which the evolution of biological systems operates: (1) $\mathrm{H}_{\mathrm{obs}}$ is an increasing function of time, as mandated by the Second Law of Thermodynamics; (2) $\mathrm{H}_{\mathrm{obs}}$ is a concave function of time, as historical constraints retard the rate of entropy increases; and (3) the difference between $\mathrm{H}_{\max }$ and $\mathrm{H}_{\mathrm{obs}}$ is an increasing function 
of time, proportional to the growth of organization in the system (Brooks and Wiley, 1988). Hence, biological systems are far from equilibrium systems and their historical constraints are responsible at least in part for their far from equilibrium status.

\section{NATURAL SELECTION}

As mentioned above, most attempts to relate information theory to biological systems have assumed that the genetic system is the source, that reproduction and ontogeny are the channel, and that the environment is the receiver. I feel that the environment cannot be a receiver, in a physical sense, because it does not measure, alter or interpret the message; it only sorts through and eliminates part of it. Therefore, the environment is a form of noise in the channel, or a filter. It plays the role of a converter rather than a receiver. Or, we can say that the environment converts some enformation (biomass or $\psi_{\mu}$ ) into intropy (dissipated energy or $\psi_{\alpha}$ ). Thus, both the growth of the information system and the elimination of information by inherent and extrinsic forces are entropic phenomena in biology.

If the environment does not act as a receiver, we must question the origin and nature (i.e., the exact role) of environmental or natural selection. We should have reason to believe that the traditional account of the reality of natural selection is incomplete, since it calls on analogy with human economic theory to explain something that is supposedly a natural process that occurred long before humans evolved. What is needed, first of all, is an account of where natural selection comes from in the first place; that is, what conditions are necessary for natural selection to occur?

The theory of natural selection is an equilibrium theory. Populations are expected to come to an equilibrium with respect to their environments, and are expected to remain at those equilibria unless the environments change. Any environmental changes create a new equilibrium point, towards which a given populations moves (adapts). How can this be reconciled with a far from equilibrium theory of evolution? The basis for the theory of natural selection was Darwin's intuition that many more offspring are produced than survive to reproduce themselves, due to limitations in necessary resources. This limitation leads to a "struggle for existence" in which only the "fittest" survive, fitness being defined by the ability to compete for limited reources, and measured by reproductive success.

To us, the key to deriving such an expectation from purely biology principles lies in understanding just what conditions allowmore organisms to be produced than there are resources available to support them.In order for there to be such "population overruns" the "rules" governing production of offspring must be 
independent of, or at least highly insensitive to, the environmental resources relevant to the offspring. Otherwise, the number of organisms produced would conform to an equilibrium number determined by the environmental resources. In addition, the offspring produced must be highly similar in needs and abilities, or they would not be expected to compete for similar resources. Thus, the autonomous production rules must be highly insensitive to environmental fluctuations and must have a high replication rate.

It is far from equilibrium systems that are characterized by autonomous production rules and by a high degree of self-organization that is manifested, in part, by the maintenance of highly organized structure. Naturally occurring populations of highly similar organisms existing a cohesive wholes are a class of steady state outcomes of far from equilibrium population dynamics. Their stability (i.e., the ability to persist through time and over space) will be proportional to their ordering due to the effects of historical processes transmitted via reproduction. This will determine the ways in which and the extent to which the population will persist in the face of changing environments.

If populations are a class of steady states, they might be expected to exist over short temporal scales during which the portion of the genealogical flow determined by the origin and spread to new information within the system will be much less that the flow determined by environmental forces. Or, in other words, populations function within boundaries defined both by history and the environment, and during the time periods that the historical effects are essentially constant, population changes will be due to environmental changes. However, the historical effects will still determine the ways in which and the extent to which each population responds to environmental changes. During these phases, populations could be treated as local equilibria (or close to equilibria) cells making up the nonequilibrium systems called species. Hence, standard population bilogical treatments, with their assumptions of equilibrium and constant (i.e., negligible) historical effects, will be useful in explaining this component of biological functioning. Natural selection and associated processes emerge from far from equilibrium views of evolution as steady state processes embedded within longterm genealogical transformations.

We may symbolize these ideas as follows. Since macroscopic information (I), or organization, increases over long time intervals (fig. 1), we expect to see an increase in the value of the function Q, the macroscopic order (Landsberg, 1984 b) or redundancy (Gatlin, 1972) of the system, where

$$
\mathrm{Q}=1-\left(\mathrm{H}_{\mathrm{obs}} / \mathrm{H}_{\mathrm{max}}\right)=\mathrm{I} / \mathrm{H}_{1}
$$


The portion of biological information that is the carrier of evolutionary constraints can be depicted as $\mathrm{H}_{\max }-\mathrm{H}_{\mathrm{obs}}$. Since this also describes macroscopic information ( $\mathrm{I}-\mathrm{H}_{\max }-\mathrm{H}_{\mathrm{obs}}$ ), biological information must be a form of physical macroscopic information. In other words, the increase in redundancy (i.e. the production of highly similar offspring) through time is accompanied by an increase in biological information in the system. Some of this information is shared between biological systems and their environment. If the environment is the source of evolutionary macroscopic organization, then the percentage of biological information that is shared with the environment should be relatively high. However, if this overlap is high, then the "fit" between biologocal systems and the environment is almost perfect; therefore, the rate of evolutionary change will be either negligible or stochastic with respect to the environment. This results in a paradoxical view of natural selection which can only be resolved by postulating that the percentage of biological information that is shared with the environment is, in fact, low. When this happens, the number of organisms requiring a particular environmental resource will exceed the availability of that resource; some organisms that are otherwise functional will not survive to reproduce or will not reproduce to the same extent as others. Thus, in order for selection to be an important evolutionary force, it must operate under conditions established by genealogically driven self-organization, which also produces constraints on the degree to which and the way in which organisms and populations can respond to natural selection.

The dependence of natural selection on organization generated by the genealogical hierarchy re-emphasizes the production component of biological systems. This production has two general features that are relevant to the question of natural selection. First, although production requires influxes of matter and energy from the environment, the rules governing the fate of that matter and energy are not found in the environment but in the organisms themselves. Production rules are thus relatively autonomous from the environment. The more autonomous the genealogical production rules are from the environment, the more likely it is that excess organisms will be produced. The primary wy to enhance the autonomy of the production rules is to increase the historical burden of constraint, so that over time the final products can be explained better by reference to their ancestry than to their current environments. This is exactly the effect that genealogical systems have. Second, the general production dynamic is one in which actual diversity increases at a slower rate than maximum possible diversity. This means that there is a high degree of redundancy in the products of genealogical processes. Working withtin this framework, selection increases redundancy by eliminating unfit variance; and thus further contributes to the increase in the macroscopic ordering of the system (fig. 3). 


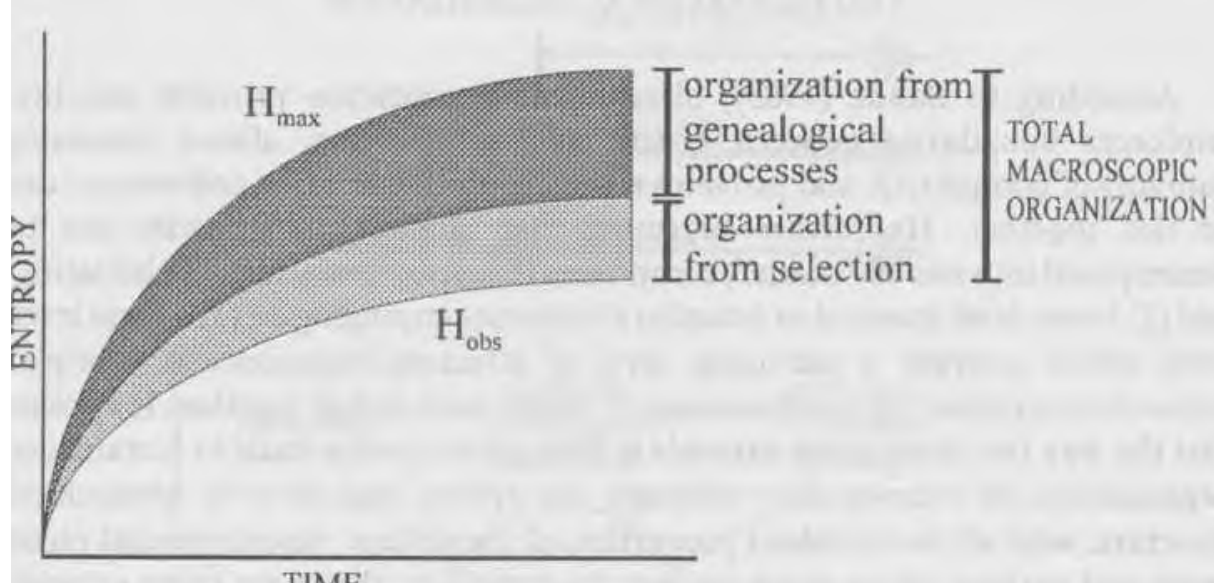

Fig. 3. The total amount of macroscopic organization $\left(\mathrm{H}_{\max }-\mathrm{H}_{\mathrm{obs}}\right)$ in biological diversity is a function of the organizing influences of genealogical processes plus the organizing influences of environmental, or selection, proecesses. These influences complement each other because their effects are to limit the increase in the entropie accumulation of diversity $\left(\mathrm{H}_{\mathrm{obs}}\right)$ over time.

\section{WHAT IS THE RECEIVER?}

Given the account of the environment as converter/noise in the channel, and the emergence of natural selection as a result of genealogical autonomy, we are left with a final question, which forms the heading of this section. I propose that the receiver is not a place, but a time. The source is the genetic system at any given to, the channel is reproduction and ontogeny, and the receiver is the genetic system at any given $\mathrm{t}_{1 \ldots \mathrm{n}}$. As the initiating conditions, the source must be ,outside” the receiver. However, there are different ways in which something can be „outside”. One of these ways is for the source to be temporally prior to the receiver. In this way, the source can produce the system (or at least encode it), and the system can later become source itself. I believe that because Darwinism, like most physical theories, is a theory of external causality, the reciver has always been construed as a part of the surroundings, i.e. a localization in space. It is true that biological systems are localized in space. The fact that all biological systems are also localized in time as well has been overlooked, or not considered imprtant. However, the temporally-dependent (spontaneously irreversible) processes in biology are all spatially-idependent. If information is dependent on a receiver, that receiver must be part of whatever is dependent for the system-in this case, time, and not space. 


\section{SCALING OF ENTROPY PRODUCTION AND HIERARCHICAL ORGANIZATION}

According to Salthe (1985), hierarchical organization provides stability, reinforces boundaries between system and surrounding, allows increasing amounts of complexity, and provides a way in which causation and control can be tied together. He further suggested that hierarchical structure can be decomposed into sets of 'triads", comprising (1) upper-level (causal or initiating) and (2) lower-level (control or boundary) elements impinging on (3) a focal level, from which emerges a particular level of structure/organization. Complex hierarchical systems are combinations of triadic units linked together. It appears that the way the phase space expands in biological systems leads to hierarchical organization of entropy flow through the system and thus to hierarchical structure, with all its attendant properties, of the system. Since potential phase space and realized phase space are not the same (i.e., the phase space expands faster than the system can fill it up) organization (biological structure) accumulates. For a given hierarchical level, then, the difference between the entropy maximum $\left(\mathrm{H}_{\max }\right)$ and the actual entropy $\left(\mathrm{H}_{\mathrm{obs}}\right)$ is proportional to the organization of the system at that level (fig. 1).

Entropy in its different manifestations is produced at different rates in biological systems because energy stored by conservative transformations is degraded at different rates. Thus, biological systems develop organized structures that exist on different spatial and temporal scales (Brooks and Wiley, 1988; fig. 4). Because of this, the parts of the evolutionary play that we can see will change depending upon the dimensions of the window through which we view it. At the lowest organizational level, the shortest time intervals, and the smallest

\section{spatial scales, the greatest relative contribution to $\psi$ will be $\psi_{\alpha}$ (external} dissipation or thermal entropy). Hence, macroscopic manifestations of $\psi_{\alpha}$ will predominate our observations in such frames of reference. For example, if we examine cellular or sub-cellular structure over short time intervals, processes such as metabolism and respiration dominate explanations of observed structure. Most entropy production is dissipated into metabolic heat loss, and the biological systems will appear to behave as classical dissipative structures. At

\section{more intermediate levels of organization, space or time, the effects of $\psi_{\mu}{ }^{b}$ are} predominant. Most entropy production at this scale is dissipated into biomass accumulation and maintenance. Finaly, on the largest and longest scales, $\psi_{\mu}{ }^{1}$

predominates, and the patterns relevant to biological explanations are formed mainly by the accumulation and maintenance of genetic diversity.

Perhaps the most important aspect of scaling considerations is the implication that there is no objective level of organization, time interval, or spatial interval for biological evolution. This is the reason Brooks and Wiley (1988) referred to their theory as a relativistic Theory. A variety of evolutionary 


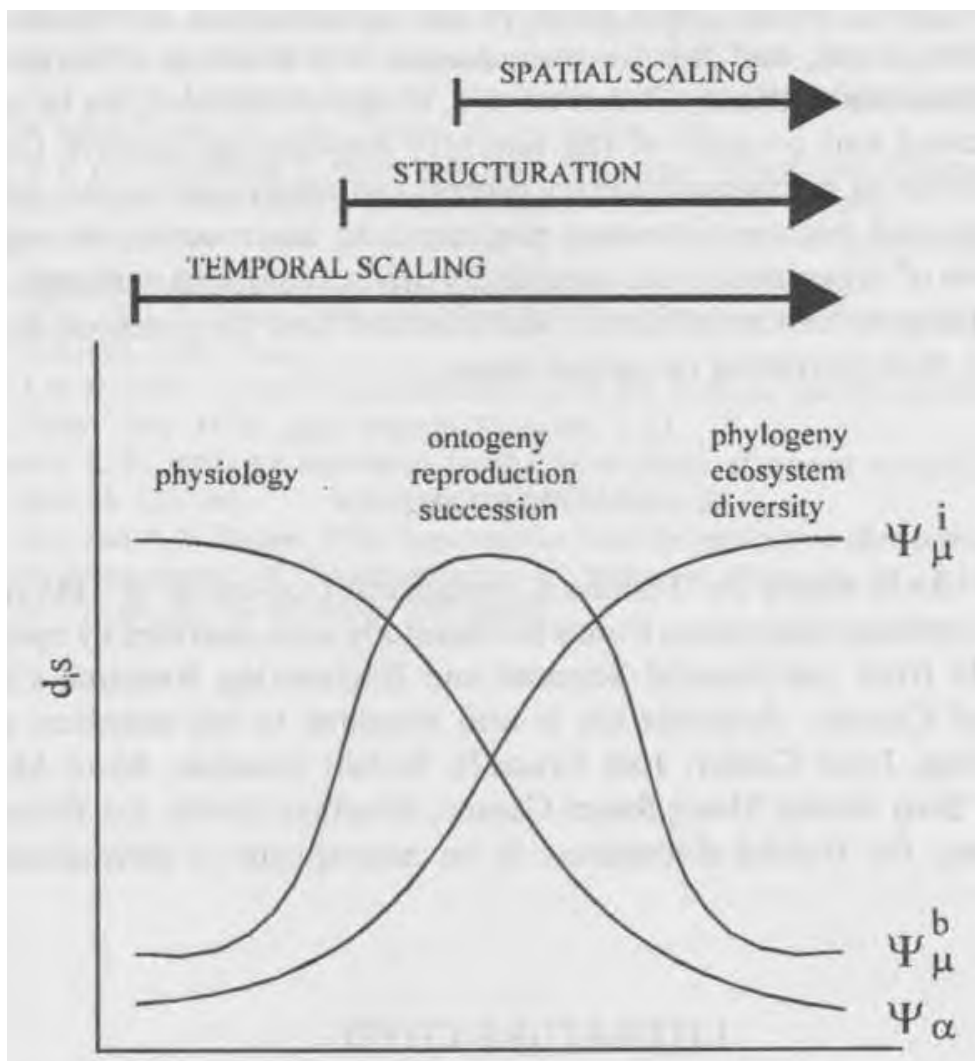

$\Delta \mathrm{t}$

Fig. 4. Temporal scaling of major production components in living systems, and their macroscopic manifestations. The axes are entropy production, $d_{S} S$, and time intervals $(\Delta t), \psi_{a}$ refers toexternal dissipation (waste), $\psi_{\mu}{ }^{b}$ to biomass accumulation, and $\psi_{\mu}{ }^{\prime}$ to genetic diversity accumulation. All processes contribute at all time intervals, but to different degrees for each $\Delta t$. This is represented heuristically by the curves accompanying $\psi_{\infty} \psi_{\mu}{ }^{b}$, and $\psi_{\mu}{ }^{1}$.

processes operate on all levels and at all scales; however, they do not all play equally important roles at all levels. Therefore, the macroscopic manifestations of evolutionary principles will differ depending on the window of observation. Microevolutionary processes, although important, are not the sole forces of evolution. They simply dominate evolution on the moderate temporal and spatial scales which are the most easily accessible windows of study for organisms with our biological and career life span constraints. I would extend his statement to include the organizing influences of entropic production on biological systems. The difference between the rate of expansion of the potential phase 
space and realized phase space leads to the accumulation of organization (biological structure), and this, in turn, creates new levels in a hierarchy of increasing structural intricacy. Based on this, bilogical evolution can be seen as the complicated end product of the interplay between the creative force of entropie increase (a macroevolutionary proces) and constraints on that increase (both macro- and microevolutionary processes). In other words, the entropie accumulation of organization and complexity drives evolutionary change, while the constraining influences of history and selection limit the potential scope of that change, thus providing its unique shape.

\section{ACKNOWLEDGMENTS}

I would like to thank Dr. Tomasz Komendzinski, co-editor of THS for the invitation to present these ideas. Funds for this study were provided by operating grant A7696 from the Natural Sciences and Engineering Research Council (NSERC) of Canada. Appreciation is also extended to the members of the AMES Group, John Collier, Joel Cracraft, Robert Geesink, Biran Maurer, Jack Maze, Stan Salthe, Doug Siegel-Causey, Jonathan Smith, Ed Wiley, and Rino Zandee, for fruitful discussions in an atmosphere of enthusiasm and cellegiality.

\section{LITERATURE CITED}

Brillouin, L. 1962. Science and Information Theory. New York: Academic Press.

Brooks, D. R., J. Collier, B. A. Maurer, J. D. H. Smith, and E.O. Wiley. 1989. Entropy and information in evolving biological systems. Biol. Philos. 4: 407-432.

Brooks, D. R., D.D. Cumming and P. H. LeBlond. 1988. Dollo's Law and the second Law of Thermodynamics: Analogy or extension? In Information Entropy and Evolution: New Perspectives on Physical and Biological Evolution, ed. B.H. Weber, D. J. Depew and J.D. Smith, pp. 189-224. Cambridge, Mass.: MIT Press

Brooks, D.R., P.H. LeBlond and D.D. Cumming. 1984. Information and entropy in a simple evolution model. J. Theor. Biol. 109: 77-93.

Brooks, D. R. and E.O. Wiley. 1988. Evolution as Entropy: Toward a Unified Theory of Biology. Chicago: Univ. Chicago Press. 2nd ed. 415 pp.

Collier, J. 1987. Entropy in evolution. Biol. Philos. 1: 5-24.

Frautschi. S.1982. Entropy in an expanding universe. Science 217: 593-599.

Frautschi, S. 1988. Entropy in an expanding universe. In Information Entropy and Evolution: New Perspectives on Physical and Biological Evolution. ed. B.H. Weber, D.J. Depew and J.D. Smith, pp. 11-22. Cambridge, Mass.: MIT Press.

Gatlin, L. 1972. Information Theory and the Living System New York: Columbia Univ. Press.

Landsberg, P.T. 1984 a. Is equilibrium always an entropy maximum ${ }^{1}$ J. Slat. Physics 35: 159-169.

Landsberg, P. T. 1984 b. Can entropy and „order” increase together? Physics Letters 102 A: 171-173.

Layzer, D. 1975. The arrow of time. Sci. Amer. 233: 56-69. 
Layzer, D. 1978. A macroscopic approach to population genetics. Z Thenr. Biol. 73: 769-788. Layzer, D. 1980. Genetic variation and progressive evolution. Amer. Nat. 115: 809-826.

Lotka, A. J. 1913. Evolution from the standpoint of physics, the principle of the persistence of stable forms. Sci. Amer. Suppl. 75: 345-6, 354, 379.

Lotka, A. J. 1925. Elements of Physical Biology. Baltimore: Williams and Wilkins.

Prigogine, I. 1980. From Being to Becoming. San Francisco: W.H. Freeman.

Prigogine, 1. and J.M. Wiame. 1946. Biologie et thermodynamique des phenomenes irreversibles. Experientia 2: 451-453.

Salthe, S. N. 1985. Evolving Hierarchical Systems: Their Structure and Representation. New York: Columbia Univ. Press.

Smith, J.D. H. 1988. A class of mathematical models for evolution and Hierarchical Information Theory. Inst. Math. Appl. Preprint Series 396: 1-13.

Ulanowicz, R. E. 1980. An hypothesis on the development of natural communities. J. Theor. Biol. 85: 223-245.

Zotin, A. 1. and R.S. Zotina. 1978. Experimental basis for qualitative phenomenological theory of development. In Thermodynamics of Biological Processes, ed. I. Lamprecht and A. I. Zotin, pp. 61-84. Berlin: DeGruyter. 